\title{
MORTALIDADE DE JOVENS POR VIOLÊNCIA NO BRASIL: DESIGUALDADE ESPACIAL E SOCIOECONÔMICA
}

\author{
Violence-related deaths among young people in Brazil: spatial and socioeconomic \\ inequality
}

\section{Mortalidad de jóvenes por violencia en Brasil: desigualdad espacial y socioeconómica}

\author{
Ana Edimilda Amador (Lattes) \\ Universidade Federal do Rio Grande do Norte - UFRN - Natal (RN) - Brasil

\section{Marilane Vilela Marques} \\ Secretaria Municipal de Saúde de Natal - SMS - Natal (RN) - Brasil \\ Marta Rovery de Souza (OrcID) \\ Universidade Federal de Goiás - UFG - Goiânia (GO) - Brasil \\ Dyego Leandro Bezerra de Souza (OrcID) \\ Universidade Federal do Rio Grande do Norte - UFRN - Natal (RN) - Brasil \\ Isabelle Ribeiro Barbosa (OrcID) \\ Universidade Federal do Rio Grande do Norte - UFRN - Natal (RN) - Brasil
}

\section{RESUMO}

Objetivo: Analisar a distribuição da mortalidade de jovens por violência no Brasil de acordo com indicadores socioeconômicos. Métodos: Trata-se de um estudo ecológico, cujas unidades de análise foram as 482 Regiões Imediatas de Articulação Urbana (RIAU) do Brasil. A variável dependente foi a Taxa de Mortalidade Padronizada (TMP) por violência em jovens (15-29 anos). Realizou-se a análise bivariada para avaliação da correlação espacial entre a variável desfecho e as variáveis independentes e a significância dos clusters. Também se aplicou o teste de correlação de Pearson, entre as variáveis. Obteve-se o número de óbitos por meio do Sistema de Informação sobre Mortalidade (SIM) e os dados da população por meio do Instituto Brasileiro de Geografia e Estatística (IBGE). Resultados: Constatou-se fraca dependência espacial entre as variáveis contextuais e a TMP nas RIAU, com valores de Moran próximos de zero. Constatou-se fraca correlação de Pearson ( $r<0,3)$. Entretanto, observou-se a formação de clusters de altas taxas de mortalidade e piores condições socioeconômicas na Região Norte e no litoral do Nordeste. Conclusão: Foi observada uma distribuição desigual da mortalidade por violência no Brasil, mesmo com fraca correlação com as variáveis socioeconômicas selecionadas.

Descritores: Mortalidade; Violência; Determinantes Sociais da Saúde.

\begin{abstract}
Objective: To analyze the distribution of violence-related deaths among young people in Brazil according to socioeconomic indicators. Methods: This is an ecological study that analyzed 482 Immediate Regions of Urban Articulation in Brazil (Regiōes Imediatas de Articulação Urbana - RIAU). The dependent variable was the Standardized Mortality Ratio (SMR) for violence among young people (15-29 years old). We carried out a bivariate analysis to assess the spatial correlation between the outcome variable and the independent variables and the significance of the clusters. Pearson's test was used to check for correlations between the variables. The number of deaths was obtained from the Mortality Information System (Sistema de Informação sobre Mortalidade - SIM) and the population data were retrieved from the Brazilian Institute of Geography and Statistics (Instituto Brasileiro de Geografia e Estatística - IBGE). Results: There was a weak spatial correlation between the contextual variables and SMR across the RIAU, with Moran values close to zero. Pearson's test found a weak correlation $(r<0.3)$. However, there were clusters of high mortality rates and worse socioeconomic conditions in the North region in the Northeastern Coastal region. Conclusion: There was an unequal distribution of violence-related mortality in Brazil despite the weak correlation to the selected socioeconomic variables.
\end{abstract}

Descriptors: Mortality; Violence; Social Determinants of Health. 


\section{RESUMEN}

Objetivo: Analizar la distribución de la mortalidad de jóvenes por violencia en Brasil según los indicadores socioeconómicos. Métodos: Se trata de un estudio ecológico cuyas las unidades de análisis fueron las 482 Regiones Inmediatas de Articulación Urbana (RIAU) de Brasil. La variable dependiente fue la Tasa de Mortalidad Estandarizada (TME) por violencia en jóvenes (15-29 años). Se realizó un análisis bivariado para evaluar la relación espacial entre la variable desfecho y las variables independientes y la significación de los clusters. También se aplicó el test de correlación de Pearson entre las variables. Se obtuvo el número de óbitos a través del Sistema de Información de Mortalidad (SIM) y los datos de la población a través del Instituto Brasileño de Geografía y Estadística (IBGE). Resultados: Se constató una dependencia espacial débil entre las variables contextuales y la TME en las RIAU con los valores de Moran cerca de cero. Se constató correlación de Pearson ( $r<0,3)$ débil. Sin embargo, se observó la formación de clusters con altas tasas de mortalidad y peores condiciones socioeconómicas en la Región Norte y en la costa del Noreste. Conclusión: Ha sido observada una distribución desigual de la mortalidad por violencia en Brasil aunque con correlación débil con las variables socioeconómicas elegidas.

Descriptores: Mortalidad; Violencia; Determinantes Sociales de la Salud.

\section{INTRODUÇÃO}

Historicamente, a violência esteve vinculada principalmente à criminalidade, objeto de estudo das ciências jurídicas e sociais. Contudo, devido às amplas dimensões que assumiu no cenário da vida moderna, esse evento tornou-se objeto multidisciplinar e interdisciplinar de estudo de outras áreas do conhecimento ${ }^{(1)}$. A morte violenta de jovens, sobretudo nos grandes centros urbanos, é um problema que vem desde a década de $1980^{(2)}$.

Não há um consenso acerca das causas associadas aos agravos violentos. São relacionados à ocorrência de morte: fatores socioeconômicos; a constituição familiar; o ambiente doméstico; a idade e as características de personalidade; o sexo da vítima; a etnia da vítima; o uso de bebidas alcoólicas e de substâncias químicas; fatores biológicos e multicausais( ${ }^{(3)}$.

A violência está inscrita e arraigada não só nas relações sociais, mas, principalmente, é construída no interior das consciências e das subjetividades. Portanto, esse fenômeno não pode ser tratado apenas como uma força exterior aos indivíduos e aos grupos ${ }^{(4)}$.

Os jovens estão imersos em contextos nos quais se manifestam distintas violências. A violência estrutural submete-os a situações de extrema desigualdade e exclusão pela falta de acesso à escola, de educação de qualidade e profissionalização, o que os impede de se integrar ao mercado de trabalho formal, possibilitando a sua inserção no mercado informal, em situações de subemprego, ou mesmo sua permanência na ociosidade ${ }^{(5)}$.

A desigualdade social refere-se a situações que implicam algum grau de injustiça, isto é, diferenças que são injustas porque estão associadas a características sociais que sistematicamente colocam alguns grupos em desvantagem com relação à oportunidade de ser e se manter sadio( ${ }^{(6)}$.

A discussão sobre a violência e suas causas torna-se imperativa no Brasil. O relatório, lançado em 2014, pela Organização das Nações Unidas (ONU), sobre o tema da prevenção global da violência apontou que $10 \%$ dos homicídios ocorridos no mundo são cometidos no Brasil. Dentre as macrocausas de homicídios no Brasil, destacamse: o crime organizado e o tráfico de drogas; a violência patrimonial; a violência interpessoal e a violência doméstica; conflitos entre policiais e população; e presença escassa do Estado nos territórios ${ }^{(7)}$.

Buscando intervir sobre a violência interpessoal, o Ministério da Saúde lançou a Portaria MS/GM $n^{\circ} 737$, de 16/05/2001, que contempla a Política Nacional de Redução da Morbimortalidade por Acidentes e Violências. Essa política trata da temática da violência como um problema social e histórico e, com isso, busca programar ações e políticas de promoção da saúde, procurando diminuir as vulnerabilidades às violências ${ }^{(8)}$.

Nisso, a Política Nacional de Promoção da Saúde, através da Portaria GM/MS n 687, de 30/03/2006, contribui com ações efetivas para a prevenção de acidentes e violências, e atua sobre os fatores de risco e de proteção, promovendo ambientes e entornos seguros e saudáveis, como também comportamentos e hábitos saudáveis por parte da população, para a redução das violências e construção de práticas solidárias e da cultura de paz ${ }^{(9)}$.

A vulnerabilidade dos jovens é tida como fator de risco para a morte violenta. Os jovens são mais suscetíveis ao aliciamento paro o tráfico de drogas, sendo uma consequência evidente que serão os mais vitimados também. Além disso, percebe-se que existe uma fragilidade específica em relação aos jovens negros ${ }^{(7)}$. 
Nesse sentido, são inúmeras as hipóteses e construções teóricas que versam sobre os determinantes da violência urbana, mas uma, em especial, chama a atenção: aquela que associa a incidência criminal urbana a formas diversas de desorganização e falta de coesão social. Essa abordagem parte do princípio de que a criminalidade emerge como consequência de problemas associados a mecanismos de controle social. Nisso, elementos que promovam a ruptura da coesão social e, consequentemente, do controle social, estariam, indiretamente, incitando práticas criminais. Tais relações seriam condicionadas por fatores sociais, como status econômico, heterogeneidade étnica, mobilidade residencial e, sobretudo, taxas de urbanização e migração(10).

Diante do exposto, o objetivo deste estudo é analisar a distribuição da mortalidade de jovens por violência no Brasil de acordo com indicadores socioeconômicos.

\section{MÉTODOS}

Trata-se de estudo ecológico de múltiplos grupos, cujas unidades de análise foram as Regiões Imediatas de Articulação Urbana do Brasil. A área deste estudo é formada pelas 482 Regiões Imediatas de Articulação Urbana (RIAU), utilizando-se como variável dependente ou resposta a mortalidade por violência, cujos óbitos constituem a somatória das categorias X85-Y09 e Y35-Y36 (Agressões - Intervenção Legal - Operações de guerra), de acordo com o capítulo XX da $10^{a}$ Revisão da Classificação Estatística Internacional de Doenças e Problemas Relacionados à Saúde (CID-10), representada pela Taxa de Mortalidade Padronizada (TMP). Para o cálculo da TMP, utilizou-se o número de óbitos na população da faixa de 15 a 29 anos, ocorridos no período de 01 de janeiro de 2001 a 31 de dezembro de 2015, distribuídos em três quinquênios (2001 a 2005, 2006 a 2010 e 2011 a 2015). Obteve-se o número de óbitos de forma secundária no Sistema de Informação sobre Mortalidade (SIM) do Departamento de Informática do Sistema Único de Saúde (Datasus).

Os dados de população por município e por idade foram obtidos das informações do Censo 2010 e das projeções intercensitárias, no sítio do Instituto Brasileiro de Geografia e Estatística. As taxas brutas foram padronizadas pelo método direto, considerando a população padrão brasileira da metade do período, e expressas por 100.000 habitantes por ano.

As variáveis independentes, representadas pelos indicadores socioeconômicos dos municípios brasileiros, foram: (V1) Índice de Desenvolvimento Humano Municipal (IDH-M); (V2) Razão de dependência; (V3) \% de crianças de 6 a 14 fora da escola; (V4) Taxa de analfabetismo de 15 anos ou mais; (V5) \% de pobres; (V6) Índice de Gini; (V7) Taxa de desocupação; (V8) \% de pessoas de 15 a 24 anos que não estudam, não trabalham e são vulneráveis na população dessa faixa; e (V9) Taxa de Trabalho Infantil. Os indicadores socioeconômicos referentes ao ano 2000 e 2010 foram coletados do Atlas de Desenvolvimento Humano no Brasil, do Programa das Nações Unidas para o Desenvolvimento (PNUD) (www.atlasbrasil.org.br).

Procedeu-se a análise descritiva das variáveis utilizadas no estudo e, para avaliar a relação entre os indicadores socioeconômicos selecionados e a TMP por violência no Brasil, foi aplicado o teste de Correlação de Pearson, considerando a significância de 0,05 . Para o processamento e a análise estatística, utilizou-se o programa IBM SPSS statistics, versão 22.0. Para análise do primeiro quinquênio, aplicaram-se as variáveis contextuais do PNUD do ano 2000, por se aproximar mais do quinquênio em estudo (2001 a 2005); no segundo (2006 a 2010) e no terceiro (2011 a 2015) quinquênios, utilizaram-se as variáveis do PNUD do ano de 2010.

Realizou-se a análise bivariada, Índice de Moran Local Bivariado (LISA), para avaliação da correlação espacial entre a variável desfecho (Taxas de Mortalidade Padronizada por violência) e as variáveis independentes. Para tanto, foram construídos os mapas temáticos com cada par de variáveis e verificados os valores de autocorrelação. Essas análises ocorreram por meio do software GeoDa, versão 1.6.61 (Spatial Analysis Laboratory, University of Illinois, Urban Champaign, Estados Unidos).

Esta pesquisa utilizou dados secundários disponíveis em sites oficiais do Ministério da Saúde do Brasil sem identificação de sujeitos, sendo dispensado de apreciação em comitê de ética em pesquisa, em conformidade com a Resolução 466/2012 do Conselho Nacional de Saúde.

\section{RESULTADOS}

A Tabela I apresenta a estatística descritiva para as variáveis dependentes e independentes. A análise descritiva dos indicadores socioeconômicos e da média da TMP por violência (100.000 hab) de acordo com o sexo, para as Regiões Imediatas de Articulação Urbana do Brasil, apresentou média da TMP, no período de 2001 a 2005, de 13.8 
para o sexo masculino e de 1.37 para o feminino. De 2006 a 2010, taxa de 16.37 para o sexo masculino e 1.53 para o sexo feminino; e, no período de 2011 a 2015, taxa de 20.05 para o sexo masculino e de 1.70 para o sexo feminino.

Assim, percebe-se pela taxa média que a mortalidade por violência no Brasil tem aumentado nos últimos 15 anos, tanto para o sexo masculino quanto para o feminino, e que a razão entre os sexos é cerca de 12:1. A análise das variáveis contextuais demonstra que o Brasil ainda apresenta baixas condições sociais e econômicas, apesar de ter apresentado uma melhora considerável em todos os indicadores nas últimas décadas.

Observa-se melhora nos indicadores no período de 2011 a 2015. O IDHM, que era de 0.56 no período de 2001 a 2005 , no período de 2010 a 2015 , foi de 0.69 . A taxa de criança fora da escola também alcançou um melhor resultado para o período de 2010 a 2015, com 2.88, e de 2005 a 2010 estava com média de 7.61 . A taxa de analfabetismo também melhorou, passando de 21.14 , no primeiro período, para 15.63 no último período do estudo. O percentual de pobres passou de 39.54 para 22.34; e a taxa de vulneráveis, de 18.50 para 14.22, em situação inversa com a taxa de trabalho infantil, em que houve uma piora, passando de 55.50 para 55.90 no último período.

A elevação do IDH, a redução de jovens fora da escola, a redução da taxa de analfabetismo e a redução do percentual de pobres demonstram o avanço social que o Brasil obteve. Todavia, ainda há a persistência de elevadas taxas de alguns indicadores, como o trabalho infantil e o número de vulneráveis à pobreza.

Tabela I - Análise descritiva dos indicadores socioeconômicos e da Taxa de Mortalidade Padronizada (TMP) por violência (100.000 habitantes), de acordo com o sexo, para as Regiões Imediatas de Articulação Urbana do Brasil. Brasil, 2018.

\begin{tabular}{|c|c|c|c|c|c|c|c|c|c|c|c|c|c|c|c|}
\hline \multirow{2}{*}{ Variáveis* } & \multicolumn{3}{|c|}{ Média } & \multicolumn{3}{|c|}{ Mediana } & \multicolumn{3}{|c|}{ Desvio padrão } & \multicolumn{3}{|c|}{ Percentis 25} & \multicolumn{3}{|c|}{ Percentis 75} \\
\hline & $1 Q^{* *}$ & $2 Q^{* *}$ & $3 Q^{*}$ & $1 Q$ & $2 Q$ & $3 Q$ & $1 Q$ & $2 Q$ & $3 Q$ & $1 Q$ & $2 Q$ & $3 Q$ & $1 Q$ & $2 Q$ & $3 Q$ \\
\hline $\begin{array}{l}\text { TMP_MASC_ } \\
\text { FEM }\end{array}$ & 7.59 & 8.92 & 10.2 & 5.53 & 6.45 & 8.48 & 6.40 & 7.56 & 8.52 & 3.17 & 3.99 & 4.67 & 9.32 & 11.77 & 14.39 \\
\hline TMP_MASC & 13.8 & 16.37 & 20.05 & 9.86 & 11.41 & 15.26 & 12.26 & 14.60 & 16.61 & 5.23 & 6.74 & 7.93 & 17.11 & 21.64 & 27.09 \\
\hline TMP_FEM & 1.37 & 1.53 & 1.70 & 1.11 & 1.26 & 1.60 & 1.15 & 1.11 & 1.07 & 0.55 & 0.79 & 0.92 & 1.82 & 2.06 & 2.31 \\
\hline IDHM & 0.56 & & 0.69 & 0.58 & & 0.70 & 0.10 & & 0.07 & 0.47 & & 0.62 & 0.64 & & 0.74 \\
\hline Dependência & 61.87 & & 51.16 & 60 & & 49.93 & 10.11 & & 7.33 & 54.12 & & 45.79 & 67.83 & & 55.32 \\
\hline Fora escola & 7.61 & & 2.88 & 6.6 & & 2.54 & 4.56 & & 1.82 & 4.86 & & 1.91 & 8.99 & & 3.22 \\
\hline Analfabetismo & 21.14 & & 15.63 & 18.76 & & 13.99 & 10.20 & & 7.89 & 12.49 & & 8.87 & 29.48 & & 22.14 \\
\hline Pobres & 39.54 & & 22.34 & 37.1 & & 18.75 & 18.68 & & 14.75 & 23.42 & & 9.03 & 57.39 & & 36.13 \\
\hline GINI & 0.54 & & 0.49 & 0.55 & & 0.49 & 0.05 & & 0.05 & 0.52 & & 0.46 & 0.57 & & 0.52 \\
\hline Desocupação & 9.54 & & 6.18 & 9.54 & & 5.94 & 3.30 & & 2.44 & 7.19 & & 4.77 & 11.52 & & 7.68 \\
\hline Trabalho infantil & 55.04 & & 55.9 & 54.82 & & 55.72 & 6.03 & & 6.6 & 50.91 & & 51.25 & 58.37 & & 59.74 \\
\hline Vulneráveis & 18.5 & & 14.22 & 19.1 & & 14.12 & 6.05 & & 6.68 & 14.31 & & 8.26 & 22.82 & & 19.63 \\
\hline
\end{tabular}

Fonte: Sistema de Informação sobre Mortalidade - SIM; Atlas Brasil, 2017. TMP: Taxa de Mortalidade Padronizada; MASC: masculino; FEM: feminino; "Para o período de 2001 a 2005, utilizaram-se variáveis contextuais referentes ao ano 2000 ; para 2011 a 2015, foram utilizadas as referentes ao ano 2010. "Período de 2001 a 2005 (1Q), 2006 a 2010 (2Q) e 2011 a 2015 (3Q); IDHM: Índice de Desenvolvimento Humano Municipal.

A correlação de Pearson entre a TMP e as variáveis socioeconômicas não apresentou forte correlação, apesar de ter sido estatisticamente significativa ao nível de 0,05 para IDHM, razão de dependência, percentual de pobres, Índice Gini, trabalho infantil e percentual de vulneráveis. Apresentou significância estatística ao nível de 0,01 para a variável taxa de desocupação. Não apresentou correlação e nem significância estatística para as variáveis: taxa de crianças fora da escola e taxa de analfabetismo. Faz-se importante destacar uma forte correção das variáveis socioeconômicas entre si, como taxa de crianças fora da escola com percentual de pobres, apresentando correlação de 0.505; e taxa de crianças fora da escola com percentual de vulneráveis, com correlação de 0.628 , além de muito forte correlação entre taxa de analfabetismo com percentual de pobres, com correção de 0.909; e de percentual de pobres com percentual de vulneráveis, de 0.931 (Tabela II).

Através da análise do Índice de Moran Local Bivariado (LISA), verificou-se fraca correlação espacial entre as variáveis socioeconômicas do estudo e a TMP por violência em jovens, apresentando valores negativos ou positivos próximos a zero. 


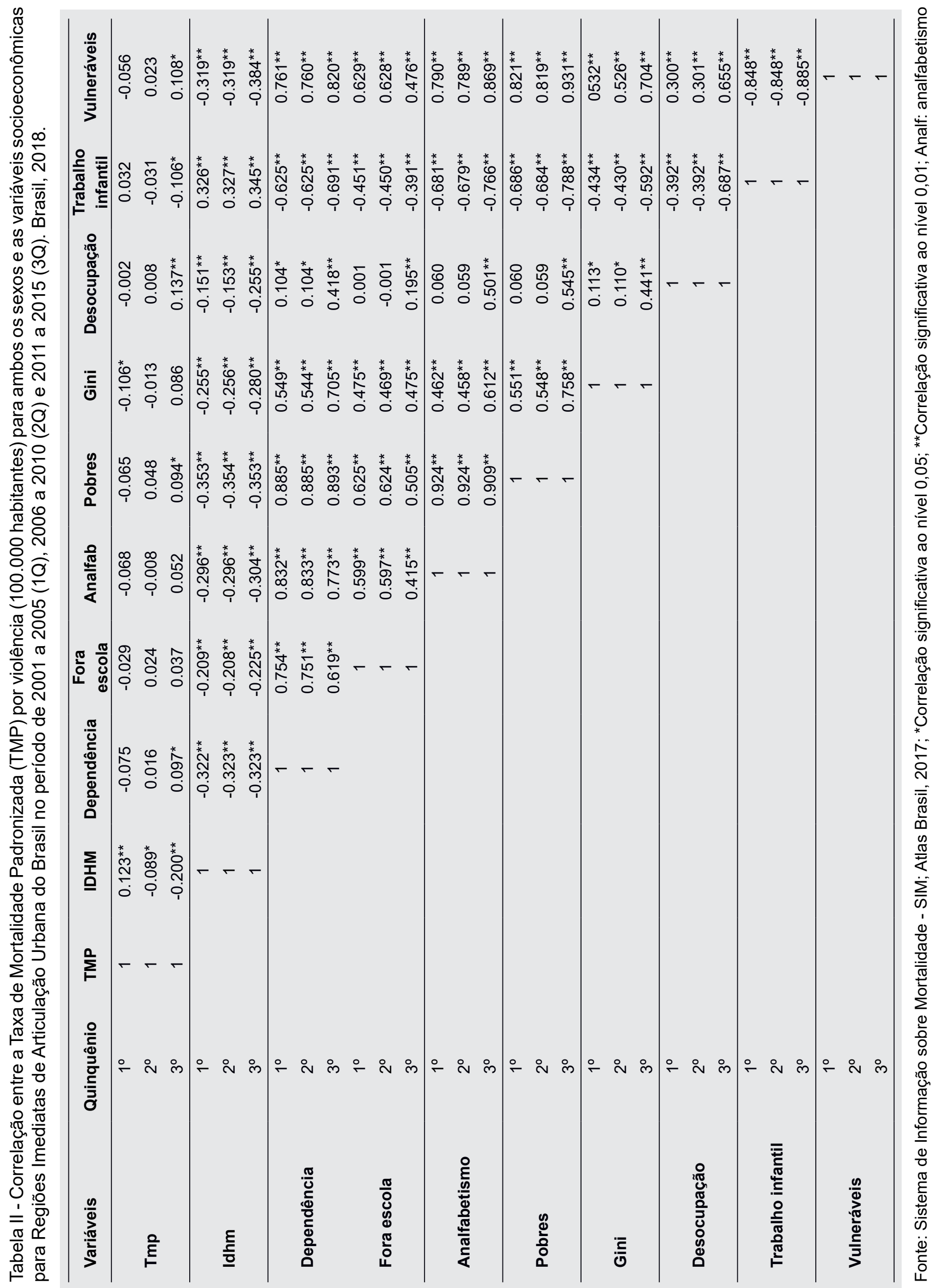


Em relação à análise espacial através do Moran bivariado entre as Taxas de Mortalidade Padronizadas por violência e as variáveis socioeconômicas das RIAU, nos três períodos (2001 a 2005, 2006 a 2010 e 2011 a 2015), observou-se formação de clusters de alto-alto, ou seja, RIAU com altas TMP e com alto percentual de pobres; assim como altas TMP em áreas com altas taxas de analfabetismo, altas TMP com alto percentual de vulneráveis e altas TMP em RIAU com altas taxas de crianças fora da escola. Esse padrão foi verificado nas regiões imediatas de articulação que se situam dentro das regiões Norte, Nordeste e Sudeste do Brasil.

\section{DISCUSSÃO}

Os resultados do presente estudo apontam que não há uma forte correlação espacial entre a violência e as condições socioeconômicas, todavia há formação de clusters de altas taxas de mortalidade e piores condições socioeconômicas nas regiões Norte, Nordeste e Sudeste do país, evidenciando que o contexto socioeconômico é um dos fatores explicativos para a distribuição da violência letal no Brasil.

Foram evidenciados os padrões de mortalidade na Região Norte do país, sendo, em grande medida, relacionados aos conflitos sociais da exploração de recursos naturais, conjugados com o avanço da fronteira agrícola. Na Região Sudeste, áreas consolidadas com altas taxas de homicídios, como as Regiões Metropolitanas do Rio de Janeiro e de Vitória, com um corredor criminal que se estende por toda a faixa litorânea fluminense ${ }^{(11)}$.

Dentre as regiões brasileiras, a Região Nordeste destaca-se por registrar, nos últimos anos, as maiores taxas de homicídio do Brasil, com uma taxa média de homicídios de 33,76 por 100 mil habitantes. Quando comparada com países com histórico de guerra civil, como o Congo $(30,8)$, e com altas taxas de homicídio associadas ao narcotráfico, como a Colômbia $(33,4)$, esses países possuem taxas menores que a do Nordeste brasileiro ${ }^{(7)}$.

Na Região Nordeste, grande parte dos municípios experimentou aumento nas taxas de homicídio, com intensificação do fenômeno em Salvador e no sul da Bahia, formado por Porto Seguro, Vitória da Conquista e Itabuna, catalisado pela expansão da atividade turística e pela recomposição da economia do cacau, que gera oportunidades econômicas, atrai migrantes, evidencia problemas sociais e acirra o potencial de conflitos. Destacam-se o entorno dos municípios maranhenses com a mancha de criminalidade, assim como o interior do Ceará e cidades litorâneas dos estados de Pernambuco e Alagoas, comandadas, respectivamente, por Recife e Maceió.

O homicídio, sobretudo o que acomete a juventude, tem sido considerado pelos estudiosos um evento marcador da violência social, embora, contraditoriamente, não seja tratado como tema prioritário pela sociedade nem pelo Estado brasileiro( ${ }^{(12)}$. Uma forma de notar as consequências da sociabilidade violenta é a falta de instâncias do Estado que ofereçam alternativas pacíficas para a mediação e a resolução de conflitos ${ }^{(7)}$, enquanto o Estado está cada vez mais voltado aos ajustes estruturais sob a preponderância do mercado financeiro, que afetam de maneira direta o mundo do trabalho e todas as dimensões da vida à medida que produz a banalização do humano(13).

Especialistas do Fórum Brasileiro de Segurança Pública apontaram que o acúmulo de vulnerabilidades sociais e a ausência de políticas públicas representam fatores de risco associados à violência homicida ${ }^{(7)}$.

Em estudo realizado em Foz do Iguaçu, Paraná, a ocupação no setor informal e a ocupação no setor formal apresentaram correlação espacial negativa $(I=-0,2574 ; P=0,0360)$ e positiva $(I=0,2574 ; P=0,0310)$, respectivamente, indicando que, quanto maior o número de empregos informais em uma determinada área, menor a taxa de homicídios nas áreas vizinhas ${ }^{(14)}$. Outro estudo realizado em Maringá, Paraná, observou que a distribuição espacial da violência se deu, predominantemente, na periferia da cidade, com os moradores de áreas pobres e com escassos serviços urbanos sendo os mais expostos à morte violenta. Em contrapartida, as classes sociais privilegiadas, residentes em lugares mais favorecidos da cidade, são as que estão mais protegidas desse tipo de violência(15).

No Brasil, no ano de 2015, o homicídio como causa de mortalidade da juventude masculina, de 15 a 29 anos, correspondeu a $47,8 \%$ do total de óbitos, uma tragédia que traz implicações na saúde, na dinâmica demográfica e, por conseguinte, no processo de desenvolvimento econômico e social( ${ }^{(16)}$. Esse fato é relevante, pois atinge uma grande parcela da população jovem em idade produtiva, trazendo consequências graves que envolvem altos custos sociais, emocionais e econômicos necessários ao tratamento e reabilitação, além de trazer danos incalculáveis para as vítimas e as famílias ${ }^{(17)}$.

A mortalidade de jovens no Brasil pode acontecer de forma seletiva. De acordo com o Relatório de Vulnerabilidade Juvenil à Violência, 2014, o panorama nacional apresenta taxa de homicídio entre jovens negros $155 \%$ maior que de jovens brancos ${ }^{(18)}$. A questão racial também tem reflexos no encarceramento brasileiro. Segundo o Mapa do Encarceramento: os jovens do Brasil, aumentou gradativamente a população negra aprisionada em relação à branca ${ }^{(19)}$. A associação entre violência, pobreza e cidadania, passando pela dimensão territorial, tem interrompido vidas de uma geração de jovens no país ${ }^{(20)}$. 
Assim, a melhoria dos indicadores sociais e econômicos registrados para o Brasil não tem sido suficiente para reduzir as taxas de homicídio no país. Em um estudo realizado no Nordeste, no período de 2001 a 2005, percebeuse que os impactos do Índice de Gini e da renda domiciliar per capita em relação às taxas de homicídio juvenil são insignificantes. Independente de, nos últimos anos, existir uma redução razoável da desigualdade de renda, sobretudo atribuída à melhoria do Índice de Gini, os homicídios permaneceram sendo praticados e, em muitos casos, tendo incremento positivo em suas taxas e números na Região Nordeste ${ }^{(21)}$.

Esse fato demonstra que o combate à violência não requer exclusiva atenção ao combate à vulnerabilidade social. Também implica a superação dos enfoques setoriais e desarticulados de grande parte das políticas sociais e de combate à violência no país. Em confluência com a desigual estrutura social e econômica do país está a ausência de esforços políticos na concretização de políticas públicas voltadas à garantia do direito à vida, revelando uma conjuntura de omissão na qual o jovem é submetido a situações violentas ${ }^{(22)}$.

Com efeito, o próprio Estado atua, em grande parte, como violador direto ou indireto do direito. A ausência de esforços políticos na concretização de políticas públicas voltadas à garantia do direito à vida revela uma conjuntura de omissão. Em confluência com esses fatores está a desigual estrutura social e econômica do País, na qual a população mais desfavorecida é vítima preferencial da violência ${ }^{(22)}$.

Especialistas do Fórum Brasileiro de Segurança Pública apontaram que o acúmulo de vulnerabilidades sociais e a ausência de políticas públicas representam fatores de risco associados à violência homicida ${ }^{(23)}$. $O$ Índice de Vulnerabilidade Juvenil (IVJ) traz uma síntese dos indicadores: taxa de frequência à escola, escolaridade, inserção no mercado de trabalho, taxa de mortalidade por causas externas, taxa de mortalidade por causas violentas, valor do rendimento familiar mensal e risco relativo de brancos e negros de serem vítimas de homicídio ${ }^{(18)}$.

No Brasil, $71 \%$ dos homicídios são perpetrados com o uso de armas de fogo, legais ou ilegais. A letalidade e o fácil acesso a esse instrumento, quando associados a uma sociedade violenta, potencializam o risco de que conflitos de ordem diversa terminem em mortes ${ }^{(7)}$. No cenário brasileiro, observa-se quase uma exclusiva masculinidade no que diz respeito ao sexo das vítimas dos homicídios por arma de fogo (HAF): 94,4\% na média nacional(24).

Em geral, as políticas no Brasil têm sido marcadas pela repressão, prisão e extermínio, sendo a juventude pobre comumente criminalizada e estigmatizada, culpabilizada e responsabilizada pela sua saúde e segurança, de tal modo a isentar as instâncias sociais e as políticas públicas como agentes de atuação frente às demandas das juventudes ${ }^{(25)}$.

Dentre os fatores associados ao crescimento da violência a partir dos anos 2000, refere-se o aumento do consumo de drogas psicoativas proibidas, em particular do crack, que ensejou o crescimento de mercados ilícitos, principalmente nos estados do Nordeste ${ }^{(26)}$. A existência de gangues e o consumo e o tráfico de drogas têm sido apontados, em diferentes perspectivas, como causadores de homicídios ${ }^{(27)}$.

Reconhecer as desigualdades sociais em saúde, buscar compreender os processos que as produzem e identificar os diferentes aspectos que estabelecem a mediação entre os processos macrossociais e o perfil epidemiológico dos diferentes grupos sociais é uma condição indispensável para que seja possível buscar formas de enfrentamento, seja no âmbito das políticas públicas, seja no âmbito da vida cotidiana ${ }^{(6)}$.

A construção de uma política efetiva de segurança pública é um pleito que se renova a cada ano. Todavia, a elaboração e implementação de políticas públicas requerem a capacidade de reunir informações para a produção de diagnósticos e indicadores que possam subsidiar o processo de formulação, execução, monitoramento e avaliação das ações governamentais ${ }^{(28)}$.

Portanto, são necessários estudos e políticas públicas voltadas aos processos que garantam os direitos de cidadania, em especial do jovem homem, negro, pobre e morador da periferia, principal vítima da violência letal no Brasil. A associação entre violência, pobreza e cidadania, passando pela dimensão territorial, tem interrompido vidas de uma geração de jovens no país. Faz-se urgente abordar as questões da desigualdade social para que, efetivamente, possa-se discutir estratégias de acesso aos direitos e à cidadania dessa geração(20).

É sabido que a violência como problema multicausal necessita de atenção intersetorial. Assim, a Política Nacional de Promoção da Saúde aponta para a necessidade de articulação com outras políticas públicas para, de forma articulada, discutir intervenções conjuntas e promover a cultura da paz ${ }^{(9)}$.

Além de articulação intersetorial e de mudanças profundas na estrutura da sociedade brasileira, é necessário trabalhar com inteligência coletiva para entender a complexa situação de violência, e pensar numa estrutura social mais igualitária e justa, com redução das iniquidades sociais. Lograr esse objetivo perpassa inclusive pelo desenvolvimento de uma cultura de paz de forma integrada, em consonância com as grandes mudanças desejadas - justiça social; igualdade entre os sexos; eliminação do racismo; tolerância religiosa; respeito às minorias; educação 
e saúde integral; equilíbrio ecológico e liberdade política. A implantação de políticas alternativas - integradoras e, sobretudo, inclusivas - dirigidas para os jovens poderia diminuir os índices de violência e, portanto, resolveria parcela considerável do problema. Entretanto, também é necessário realizar uma profunda reflexão sobre os valores, as formas de socialização e a construção das identidades masculina e feminina nas sociedades atuais.

A limitação deste estudo está relacionada à utilização de dados secundários sobre mortalidade, que estão sujeitos ao subregistro, apesar de, nos últimos anos, reconhecer-se que o Sistema de Informação sobre Mortalidade (SIM) no Brasil obteve ganhos significativos quanto à qualidade da informação. Outra limitação refere-se ao delineamento do estudo, pois a possibilidade do viés ecológico é sempre lembrada como uma limitação para o uso de correlações ecológicas.

As contribuições deste estudo, por sua vez, referem-se aos subsídios gerados para políticas públicas com ênfase nas ações de promoção e prevenção territorialmente determinadas, que poderão ser usadas como critério para repartição mais equânime dos recursos públicos, priorizando as regiões que apresentam os piores indicadores.

Os resultados do presente estudo facilitam a compreensão das características da violência no Brasil e contribuem com subsídios para que sejam direcionadas políticas públicas intersetoriais e para que as propostas de intervenção sejam pautadas em diagnósticos que levem em consideração as características específicas de cada região, priorizando as regiões que apresentam os piores indicadores, de forma que essa priorização venha a prevenir perdas evitáveis.

\section{CONCLUSÃO}

A distribuição espacial da violência homicida tem se revelado fonte profícua de descobertas para a análise dos fatores que incidem em sua produção e reprodução. Este estudo permitiu identificar importantes características relacionadas às mortes por violência, bem como mudanças nos padrões de distribuição espacial verificados nos 15 anos analisados. Observa-se uma distribuição desigual da mortalidade por violência no Brasil, mesmo com fraca correlação com as variáveis socioeconômicas selecionadas.

\section{REFERÊNCIAS}

1. Gawryszewski VP, Kahn T, Mello Jorge MHP. Informações sobre homicídios e sua integração com o setor saúde e segurança pública. Rev Saúde Pública. 2005; 39(4):627-33.

2. Ruotti C, Almeida JF, Regina FL, Massa VC, Peres MFT. A vulnerabilidade dos jovens à morte violenta: um estudo de caso no contexto dos "Crimes de Maio". Saúde Soc. 2014;23(3):733-48.

3. Martins CBG, Jorge MHPM. Óbitos por causas externas em Cuiabá, 0 a 24 anos: perfil das vítimas e famílias segundo a intencionalidade. Rev Bras Epidemiol. 2013;16(2):454-68.

4. Minayo MCS. A Violência Social sob a Perspectiva da Saúde Pública. Cad. Saúde Públ., 1994;10(Supl 1):S7S18.

5. Silva JG. Homicídio de jovens no Brasil: o desafio de compreender a consequência fatal da violência [tese]. Rio de Janeiro: Escola Nacional de Saúde Pública Sergio Arouca; 2014.

6. Barata RB. O que queremos dizer com desigualdades sociais em saúde? In: Como e por que as desigualdades sociais fazem mal à saúde. Rio de Janeiro: FIOCRUZ; 2009. (Temas em Saúde collection). p. 11-21.

7. Ministério da Justiça (BR). Diagnóstico dos homicídios no Brasil: subsídios para o Pacto Nacional pela Redução de Homicídio. Brasília: Ministério da Justiça; 2015.

8. Patrício AP. O papel da violência interpessoal compreendida como a manifestação de uma endemia social: uma análise geográfica sobre o fenômeno no Brasil e Rio Grande do Norte [dissertação]. Natal: Universidade Federal do Rio Grande do Norte; 2014.

9. Ministério da Saúde (BR), Secretaria de Vigilância em Saúde, Secretaria de Atenção à Saúde. Política Nacional de Promoção da Saúde: PNPS: revisão da Portaria MS/GM n 687, de 30 de março de 2006. Brasília: Ministério da Saúde; 2015.

10. Diniz AMA. Migração, desorganização social e violência urbana em Minas Gerais. Rev RA’EGA. 2005;9:9-23.

11. Waiselfisz JJ. Mapa da violência 2011: os jovens do Brasil. Brasília. Instituto Sangari; 2011. 
12. Costa DH, Schenker M, Njaine K, Souza ER. Homicídios de jovens: os impactos da perda em famílias de vítimas. Physis (Rio de J). 2017;27(3):685-705.

13. Guilherme RC, Reis CN. Plano Estratégico de Ação Social do MERCOSUL: a transferência de renda em contexto de ofensiva neoliberal. Rev Katálysis. 2018;21(1):108-24.

14. Andrade L, Nihei OK, Pelloso SM, Carvalho MDB. Homicídios juvenis e informalidade em um município brasileiro da tríplice fronteira Brasil, Paraguai e Argentina. Rev Panam Salud Publica. 2012;31(5):380-7.

15. Fonzar UJV, Soares DFPP, Santil FLP. Espacialização das três principais causas de morte no município de Maringá, Estado do Paraná, em 1996. Acta Sci Health Sci. 2002;24(3):765-74.

16. Cerqueira D, Lima RS, Bueno S, Valencia LI, Hanashiro O, Machado PHGM, et al. Atlas da Violência 2017. Rio de Janeiro: IPEA; 2017 [acesso em 2018 Maio 7]. Disponível em: http://ipea.gov.br/atlasviolencia/

17. Sousa ASB, Silva SC, Cavalcante MFA. Mortalidade por causas externas em adultos jovens em Teresina-PI no período de 2001-2011. Rev Interd. 2016;9(1):57-65.

18. Secretaria Geral da Presidência da República (BR), Secretaria Nacional de Juventude, Ministério da Justiça. Índice de vulnerabilidade juvenil à violência e desigualdade racial 2014. Brasília: Presidência da República; 2015. (Série Juventude Viva).

19. Barbosa KS, Coelho NMMS. A questão étnico-racial do sonho americano: o encarceramento dos pobres e negros no Estado policial. Rev Bras Segur Pública. 2017;11(1):164-82.

20. Medeiros TJ, Malfitano APS. Entre a violência e a cidadania: um olhar sobre a mortalidade juvenil. Mundo Saúde. 2015;39(1):62-73.

21. Nobrega JM. A queda da desigualdade de renda no Brasil e os homicídios na Região Nordeste. Rev Espaço Acadêmico, 2009;9(98):72-80.

22. Malta DC, Minayo MCS, Soares AM Filho, Silva MA, Montenegro MMS, Ladeira RM, et al. Mortalidade e anos de vida perdidos por violências interpessoais e autoprovocadas no Brasil e Estados: análise das estimativas do Estudo Carga Global de Doença, 1990 e 2015. Rev Bras Epidemiol, 2017; 20 Supl 1:142-56.

23. Câmara dos Deputados (BR). Relatório Final Comissão Parlamentar de Inquérito Homicídios de Jovens Negros e Pobres. Brasília: Câmara dos Deputados; 2015 [acesso em 2018 Maio 7]. Disponível em: http://www.camara. gov.br/proposicoesWeb/prop_mostrarintegra?codteor=1361419

24. Waiselfisz JJ. Mapa da Violência 2016: homicídios por armas de fogo no Brasil. Brasília: FLACSO; 2016.

25. Sinhoretto J. A filtragem racial na seleção policial de suspeitos: segurança pública e relações raciais. In: Figueiredo I. Segurança pública e direitos humanos: temas transversais. Brasil: Ministério da Justiça; 2014. p. 121-59.

26. Cerqueira D. Causas e consequências do crime no Brasil [tese]. Rio de Janeiro: PUC; 2014.

27. Waiselfisz J. Mapa da Violência 2014: os jovens no Brasil. Brasília: FLACSO; 2014.

28. Figueira MG. Proposta de uma matriz de indicadores para as ações de gestão de informação da SENASP. Rev Bras Segur Pública. 2015;9(2):110-28.

\section{Endereço para correspondência:}

Ana Edimilda Amador

Universidade Federal do Rio Grande do Norte - UFRN

Av. Senador Salgado Filho, 3000

Bairro: Lagoa Nova

CEP: 59078-970 - Natal - RN - Brasil

E-mail: edimilda-amador@hotmail.com 